\title{
The puzzle of the steady-state rotation of a reverse sprinkler
}

\section{Citation}

Rueckner, Wolfgang. 2015. "The Puzzle of the Steady-State Rotation of a Reverse Sprinkler." American Journal of Physics 83 (4) (April): 296-304. doi:10.1119/1.4901816.

\section{Published Version}

10.1119/1.4901816

\section{Permanent link}

http://nrs.harvard.edu/urn-3:HUL.InstRepos:27413727

\section{Terms of Use}

This article was downloaded from Harvard University's DASH repository, and is made available under the terms and conditions applicable to Other Posted Material, as set forth at http:// nrs.harvard.edu/urn-3:HUL.InstRepos:dash.current.terms-of-use\#LAA

\section{Share Your Story}

The Harvard community has made this article openly available.

Please share how this access benefits you. Submit a story.

Accessibility 


\title{
The puzzle of the steady-state rotation of a reverse sprinkler
}

\author{
Wolfgang Rueckner ${ }^{\text {a) }}$ \\ Harvard University Science Center, Cambridge, Massachusetts 02138
}

(Received 21 August 2013; accepted 3 November 2014)

\begin{abstract}
The continuous rotation of the reverse sprinkler has been a puzzle for over two decades. This article presents a series of experiments that demonstrate that a properly designed reverse sprinkler experiences no steady-state torque and does not rotate. Ignoring transients when the flow starts and stops, if any sustained rotation of the reverse sprinkler occurs, it is because a force couple produces a torque accompanied by vortex flow inside the body of the sprinkler. No steady-state rotation occurs if the vortex is suppressed or prevented from forming in the first place. Demonstrative proof is given that an ideal reverse sprinkler does not rotate. (c) 2015 American Association of Physics Teachers.

[http://dx.doi.org/10.1119/1.4901816]
\end{abstract}

\section{INTRODUCTION}

A sprinkler connected to a source of compressed air will rotate opposite to the direction of the air leaving the nozzles, as one intuitively expects. A question one might ask is, "what direction will the sprinkler rotate if connected to a vacuum pump (reverse mode) instead of a compressed air source (normal mode)?" The reverse sprinkler is a seemingly simple physics problem that received much attention in the late 1980s after Richard Feynman's account of his own experimentation with it. ${ }^{1}$ Several articles appeared in this journal, ${ }^{2-4}$ some disagreeing with each other on what actually happens. The demonstration teams at Harvard and the University of Maryland ${ }^{5}$ entered the fray in hopes of settling the question. Although different in design as well as fluid employed (air and water, respectively), both versions turned in a direction toward the incoming fluid. A detailed kinematic study of both our air and their water sprinkler was subsequently published by Collier et al., ${ }^{6}$ in which they concluded that their reverse-water sprinkler rotates with an angular momentum opposite to and approximately equal to that of the fluid in the azimuthal, or tangential, section of the sprinkler arms. As for the air sprinkler, their conclusion was that one must consider the effects of both turbulence and the motion of the surrounding air-in short, dissipative effects are significant enough so that conservation of angular momentum for just the sprinkler does not hold. If angular momentum conservation were to hold, it would be impossible for the rotational frequency of the sprinkler head to increase without a corresponding increase in the velocity profile of the surrounding fluid.

A little more than a decade later, Jenkins ${ }^{7}$ offered an elementary and excellent treatment of the reverse sprinkler problem, suitable for introductory physics classes. Readers interested in a comprehensive historical review are encouraged to consult his paper. He argues that the sprinkler experiences no net torque to make it rotate except for transients when the fluid starts and stops flowing; it briefly moves toward the incoming fluid at the start and in the opposite direction when the fluid flow stops. The fact that the reverse sprinkler continues to rotate in the interim may be attributed to dissipative effects.

What has been lacking in all these years is a satisfactory explanation of what these dissipative effects really are and how they produce steady-state rotation. This motivated a systematic examination of the sprinkler to identify the underlying mechanism responsible for rotation. In the present article, we report on these experiments and conclude that vortex rotation of fluid in the body of a reverse sprinkler is the main factor that determines whether it will exhibit steady-state rotation.

\section{BACKGROUND}

Figure 1 shows a drawing of the sprinkler that has been used as a lecture demonstration for the past $26 \mathrm{yr}$. To minimize friction, a novel method of coupling the tube supplying air or vacuum to the sprinkler was employed. The coupling consists of two concentric tubes: an outer tube, fixed to the apparatus base, is connected to either the air or vacuum supply hose; and an inner tube, fixed to the rotating hub of the sprinkler. The tubes are adjusted so that there is very little clearance between them $(0.25 \mathrm{~mm})$. Unavoidably, some gas leaks through the space between the tubes, but that small leakage is more than compensated by the reduction in friction due to the absence of a rotating coupling.

The sprinkler's rotation is unmistakable and reliablewhen operated in the reverse mode, it rotates opposite to the

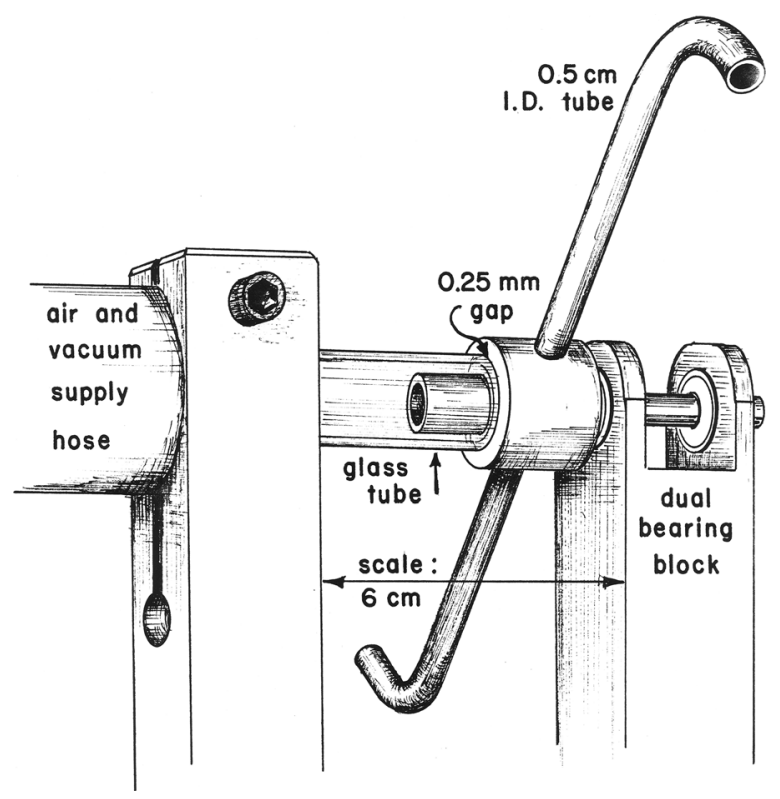

Fig. 1. Drawing of original sprinkler at Harvard still in use today. There is a $1 / 4$-mm gap between the air/vacuum supply and the sprinkler hub to provide a coupling without contact. 
direction of rotation in the normal mode, albeit eight times more slowly. It does not matter whether air is "sucked" into the sprinkler by a vacuum or "pushed" into the sprinkler by an external high-pressure source; a pressure difference between the inside and outside of the sprinkler is all that matters. We verified this by placing the sprinkler in a sealed aquarium (without water) fitted with an air inlet to pressurize the sprinkler's environment, and an air outlet for the hose that is normally connected to a vacuum. Using this method of operation, the reverse sprinkler rotated in the reverse direction just as well as the vacuum method.

The rotation is not a transient phenomenon. Starting from rest, the sprinkler accelerates to its terminal rotational speed in the reverse direction. If it is stopped and given a spin in the opposite (normal) direction, it will slow to a stop, reverse direction, and accelerate back to its original terminal angular velocity. If the reverse rotation were due to only transient effects, then no net torque would be expected under steady flow conditions. Because it rotates under steady flow conditions, there is a net torque.

Originally we attributed the motion to viscosity, arguing as follows. For a non-ideal fluid, viscosity and turbulence would have the effect of dragging more fluid toward the entry nozzle of the sprinkler than actually enters the nozzle. Thus, part of the fluid's momentum is owned by the surrounding fluid and, in turn, its surroundings. To conserve the total angular momentum, the angular momentum of the sprinkler arm should be equal to the angular momentum of the fluid entering the nozzle plus that of the surrounding fluid. Thus the sprinkler's angular momentum would be slightly larger and opposite to the angular momentum of the fluid inside it, causing it to rotate. Our viscosity argument lost steam when put to the test in 1991. To maximize the viscosity, the sprinkler was put in an argon environment instead of air; the viscosity of argon is about $27 \%$ greater than that of air or $\mathrm{N}_{2}$. The viscosity of argon varies with temperature $^{8}$ and thus we were able to measure its effect by changing the temperature. A $12 \%$ decrease in viscosity produced a $6 \pm 3 \%$ decrease in rotational speed. Since we did not know if and how much the temperature change affected the bearings, the data points were not statistically convincing enough to further pursue this avenue of investigation. A few years later, we tried to literally see if any fluid "overshoots" the nozzle when being sucked in. A mixture of water and rheoscopic fluid was sucked out of a tank through a $1 / 4$ in.-diameter tube. Absolutely no overshoot was visible to the eye.

\section{PRESENT INVESTIGATION}

To get to the bottom of what makes the reverse sprinkler rotate in the steady-state condition, a new design was in order. Essential to the new design was the ability to easily change parameters to determine which ones affect the rotation and to what extent. Specifically, (1) the length of the tangential intake nozzle, (2) the radius of the bend in the nozzle, and (3) the manner in which the fluid leaves the sprinkler were three parameters of interest.

A lightweight acrylic turntable on low-friction bearings was fabricated and mounted on an optics rail (see Fig. 2). Attached to the bottom of the turntable is a cardboard disk with a hole near its edge. This provides a means to accurately monitor the rotation frequency with an IR photo-detector/frequency counter combination.

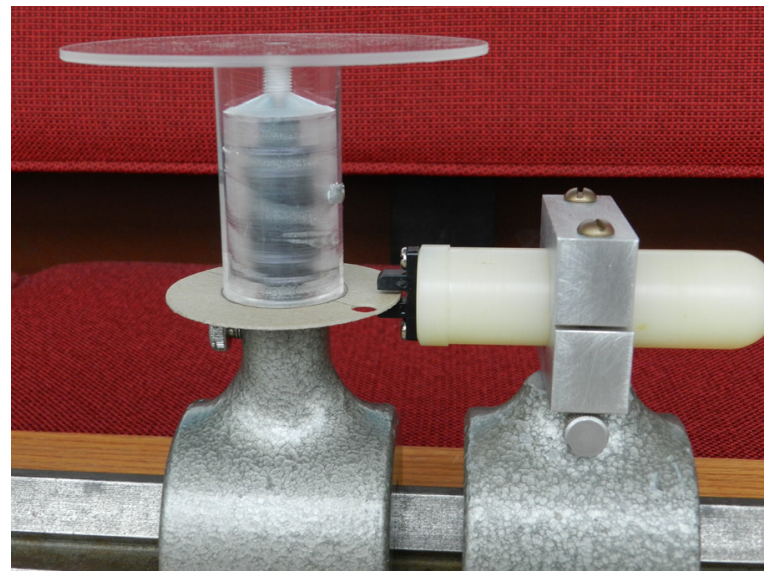

Fig. 2. Acrylic turntable for holding sprinkler assemblies. An IR photo-gate at the bottom monitors the rotation frequency.

Various sprinkler assemblies can be attached to the turntable; the entire set-up is shown in Fig. 3. In this case, the sprinkler assembly consists of two soda straws fashioned to the sides of an 8-oz plastic deli container. The container's lid attaches to the turntable and a hole in the bottom of the container provides a means of sucking the air out.

Minimizing friction was still paramount and the new design utilizes the same principle employed in the original air hose/sprinkler coupling. That is, the exhaust hose is coupled to the sprinkler without physically touching it-a fine screw adjustment (shown on the left in Fig. 3) allows one to move the hose adapter as close as possible to the exhaust hole in the sprinkler to minimize the air gap.

In addition, plastic "slim" straws (3.6 mm I.D. $\times 70 \mathrm{~mm}$ long) were packed into the hose adapter to minimize the rotation of air inside the exhaust hose, the assumption being that such a rotation would have the effect of adding angular momentum to the sprinkler assembly (see Fig. 4).

In comparing the effects of changing sprinkler parameters, it is important to be able to change one at a time while keeping the others constant. For example, it is desirable to maintain a constant airflow through the sprinkler. An airflow monitor was devised from a 2 in. muffin fan and attached to the output of the air source. The muffin fan acts like an electric generator with an output voltage of $19 \mathrm{VAC}$ at maximum airflow. With the air source powered through a Variac, ${ }^{\mathrm{TM}}$ the air flow can be adjusted for some desired monitor voltage,

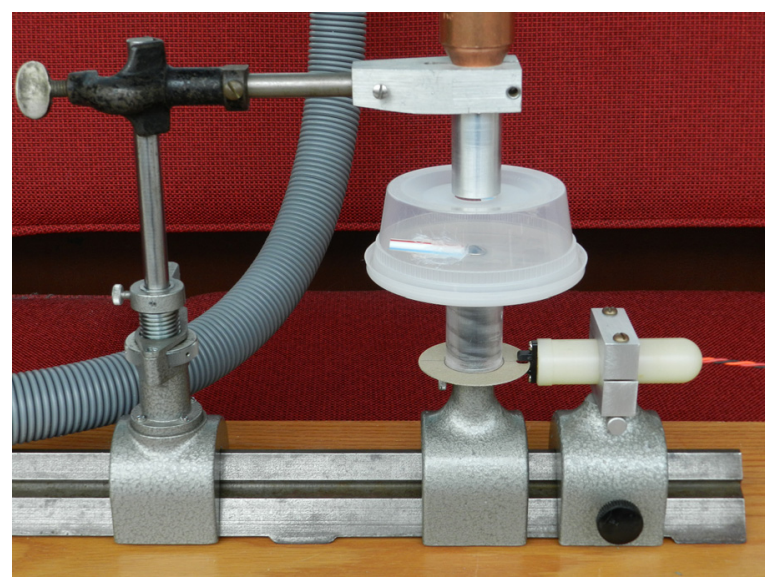

Fig. 3. The entire set-up with deli container sprinkler in place. 


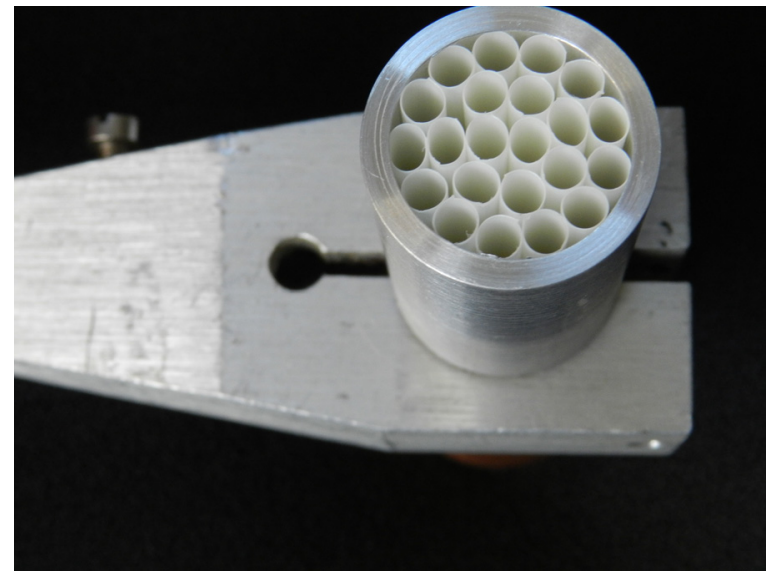

Fig. 4. Exhaust hose coupler packed with small straws to minimize rotation of air as it is removed from sprinkler assembly.

making it possible to maintain the same flow rate, albeit uncalibrated, for various sprinkler designs.

The first parameter to be tested was the length of the sprinkler's intake nozzle. How might the length affect the rotation rate? Since the incoming air experiences a prolonged acceleration inside a longer intake nozzle, the impulse/momentum theorem would suggest (incorrectly, it turns out) that the momentum of the sprinkler would be greater for longer nozzles. Five sprinkler assemblies were made with straws ranging from 1 in. to 4 in. long, as shown in Fig. 5.

The nozzle straws were mounted so that the air enters tangentially to the containers' walls. The idea is to try to minimize the torque on the sprinkler due to the reaction force of the air on the wall. Unlike an L or S-shaped sprinkler, in which the air is deflected inward by the bend in the sprinkler arm, in this geometry the wall is not responsible for the inward deflection of the air. It is expected that, due to the viscosity of the air, some drag forces on the wall are unavoidable but would be relatively small compared to wall forces in an L-shaped sprinkler.

Minimizing the reaction force of the air on the wall had a remarkable effect: not only did the sprinkler rotate towards the incoming air, the rotation frequencies were surprisingly high-the largest being $115 \mathrm{~Hz}(6900 \mathrm{rpm})$ ! The results are

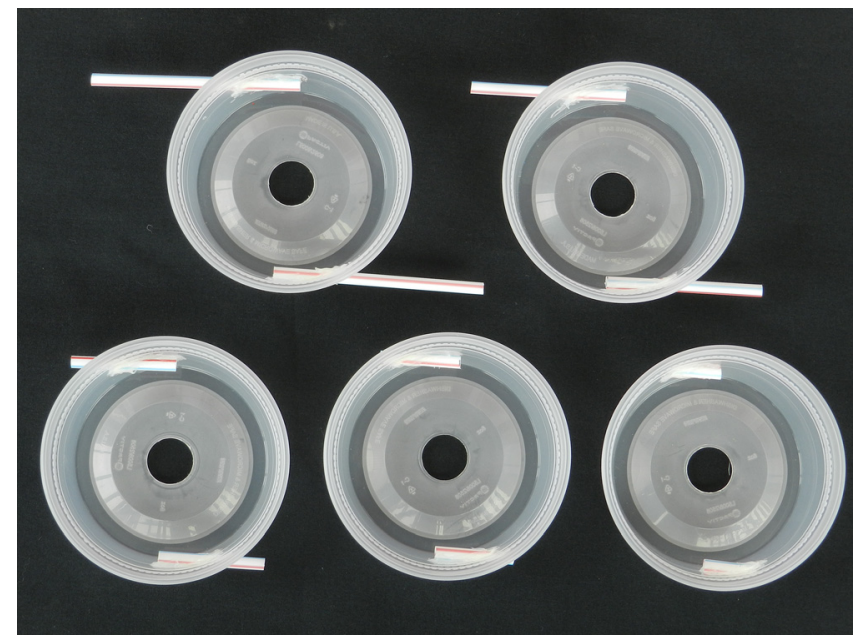

Fig. 5. Five sprinkler assemblies with nozzle straws ranging from 1 in. to 4 in. long. plotted in Fig. 6. It is clear that the nozzle affects the rotation speed adversely: the longer the nozzle, the smaller the rotation speed. The graph shows an inverse relationship up to a point; nozzles less than $11 / 2$-in. long ( 0.66 in. $^{-1}$ on the graph) have much less of an effect on the speed of rotation. One can surmise that increasing the length of the nozzle proportionately increases the drag force on the sprinkler assembly as the nozzle arm sweeps through the surrounding air. Shortening the nozzle length proportionately reduces the drag until it is less than the overall friction, at which point shortening further makes little difference. The graph levels off to a maximum rotational frequency of about $115 \mathrm{~Hz}$, limited by the overall drag of the surrounding air on the apparatus as well as the turntable bearings.

One of the reviewers of this manuscript suggested overcoming the air drag by positioning the various length nozzles on the inside instead of the outside of the containers. To that end, a large $(16 \mathrm{~cm})$ diameter container was used to accommodate the various length straws on the inside. The rotation rates were much smaller for this large container. Nevertheless, the rotation rates also increased with a decrease in nozzle length, although not as dramatically. The increase was about $30 \%$ between the longest (4-in.) nozzle and the shortest (1-in.) nozzle. The air inside the container is in motion and consequently these nozzles also experience air drag. However, it is not as pronounced because the nozzle straws are "swept back" with respect to the moving air and offer a more streamlined aerodynamic profile compared to the nozzles on the outside. Whether on the outside or inside, the drag force on the nozzle arm has a much greater effect than the length of time the air accelerates inside the nozzle. The latter seems to have no noticeable effect on the angular momentum and speed of rotation.

One can also surmise that the air circulating inside the sprinkler must constitute a strong vortex before it is sucked out through the exhaust hole. The notion of air circulating inside a cylindrical vessel is not new. For example, Ernst Mach explained a similar experiment in 1883 as follows (see Fig. 7): "If we place a hollow cylinder on a pivot, after the side has been slit and bent in the manner indicated, the cylinder will turn in the direction of the long arrow when blown into and in the direction of the short arrow when sucked on. The air, here, on entering the cylinder, can continue its rotation unimpeded, and this motion is accordingly compensated for by a rotation in the opposite direction." $9 \mathrm{We}$ interpret

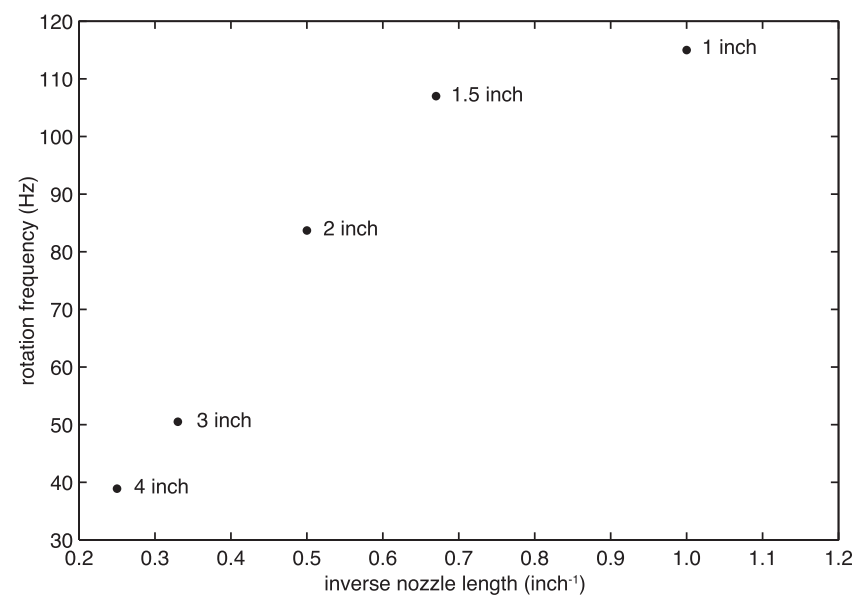

Fig. 6. Inverse relationship between rotation frequency and nozzle length. Annotations on data points indicate nozzle length. Shorter nozzles increase rotation up to a point. 


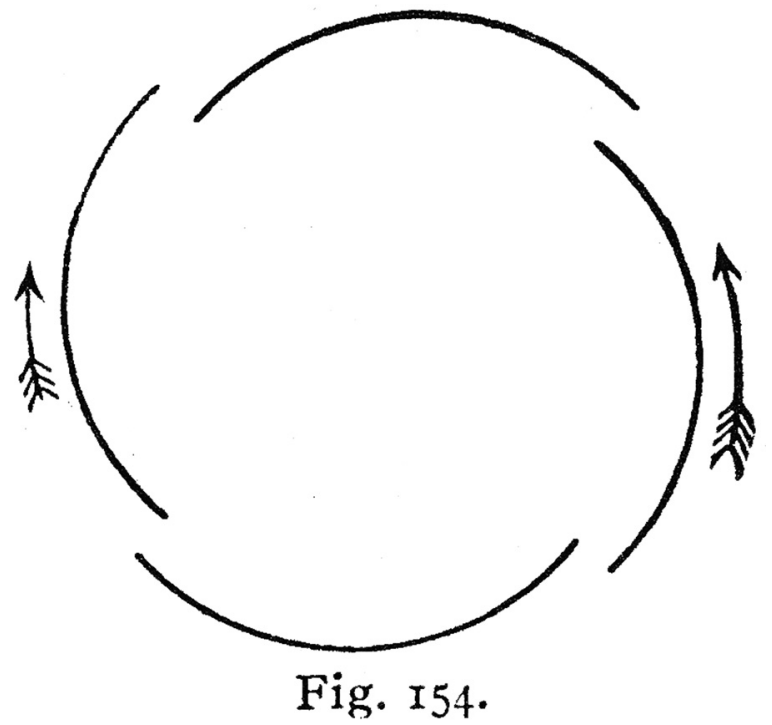

Fig. 7. Mach's cylinder experiment (see Ref. 9).

"the motion is accordingly compensated" as a statement of conservation of angular momentum. Figure 7 is a reproduction of his Fig. 154.

More recently, Carl Mungan described experiments he performed using a soda can with various drinking straw nozzle configurations (see Fig. 8). ${ }^{10}$ When the can is partly submerged in a sink full of water, water flows into the can through the straw nozzles. He reports small and transient rotations and attributes them to the angular momentum carried by the water (relative to the axis of the can) as it enters the can, and affirms that Jenkins' analysis (Ref. 7) correctly predicts the behavior for the various nozzle configurations. He concludes with a concept question, intended to be used by instructors: ask the class to predict the directions of rotation of the sprinkler in both the forward and reverse flows.

Intrigued, we decided to try out these variations ourselves. Instead of soda cans, three quart-sized plastic containers were outfitted with intake nozzles near their bottom as shown in Fig. 9.

When placed in a sink full of water, the containers sank down as water flowed in (reverse mode). The circulating flow of the water inside the container was made visible by adding a pinch of black pepper flakes as well as floating tiny (1-3 mm diameter) Styrofoam balls on the surface. The container with the $\mathrm{U}$-shaped nozzles rotated $\mathrm{CW}$ with a period of approximately $10 \mathrm{~s}$ per rotation, while the water inside could be seen to rotate in the opposite direction at about twice that rate. Unlike Mungan's transient rotations, these rotations were continuous. Furthermore, if the container was stopped momentarily and then released again, it would again continue its rotation opposite to the water rotation. The container with S-shaped nozzles rotated CCW with about half the speed of the container with the U-shaped nozzles, while the water inside rotated in the opposite direction. The container with L-shaped nozzles did not rotate and the water inside exhibited random motion. On rare occasions, it would rotate very slowly in either direction, accompanied by water rotating in the opposite direction.

These results raised the question of whether or not the original reverse water sprinkler at the University of Maryland (Ref. 5) behaves similarly. The central hub of the
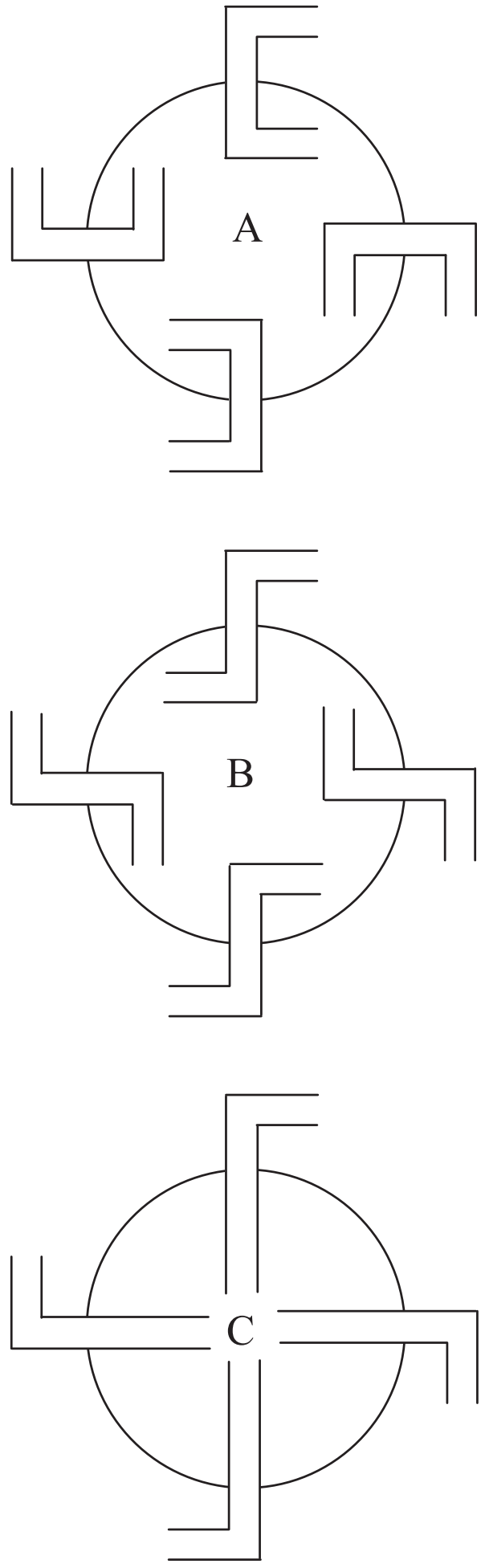

Fig. 8. Carl Mungan's concept question: predict the direction of rotation in each of the three cases. (Reproduced with permission from C. Mungan, "Inverse lawn sprinkler," Phys. Teach. 43, L-1-L-2 (2005). Copyright 2005, American Association of Physics Teachers.)

UMD sprinkler is by far the largest part of their assembly, very similar to the container with L-shaped nozzles in Fig. 9. To operate the L-shaped nozzle sprinkler in a pressurized environment (duplicating the UMD conditions), the plastic container was sealed closed with its cover. The sprinkler assembly was placed in a $81 / 2$-in. diameter cylindrical vessel that was half filled with water, and that, in turn, was put 


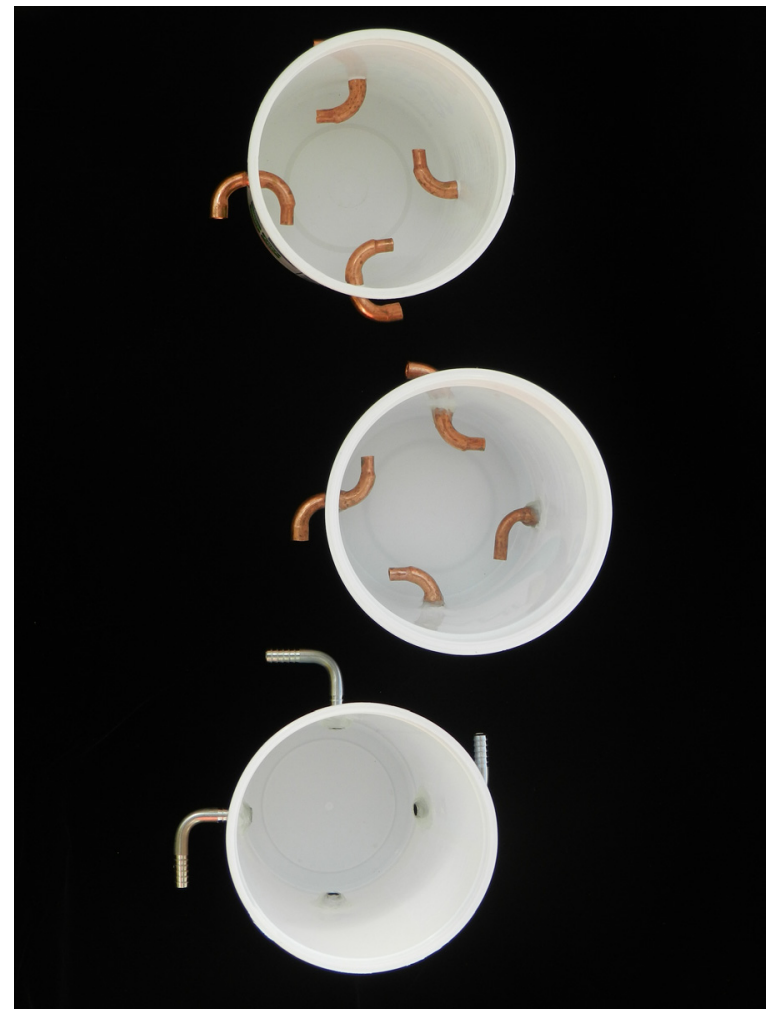

Fig. 9. A realization of Mungan's concept question.

inside a $10^{1 / 4}$-in. diameter bell jar. Using a gas regulator, the bell jar was pressurized with nitrogen gas to approximately 2 psi, which forced water into the sprinkler. Unlike the UMD sprinkler, this sprinkler did not turn in the reverse direction but instead in the same direction as the water entering the nozzles; i.e., it "looked" like a normal sprinkler. This was not surprising as we have found that the direction of water rotation, if there is any rotation at all, is random and determined by some subtle asymmetry. To validate this idea, aluminum window screening was put inside the hub, as shown in Fig. 10.

Indeed, the screening prevented the formation of a vortex inside the hub and the sprinkler did not rotate. The UMD mathematical model of the sprinkler predicts a rotation of the sprinkler due to water moving in the azimuthal section of the nozzles; it is that which contributes to the angular momentum of the system. Since their mathematical model only

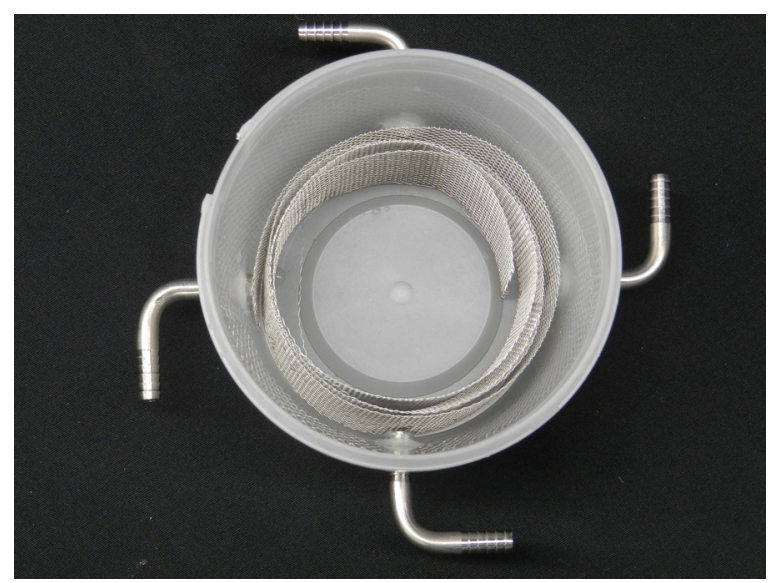

Fig. 10. Aluminum window screening inside hub of water sprinkler. dealt with the sprinkler arms, they must have assumed that the water entering the hub randomly mixes inside and does not contribute to the motion. The present experiment demonstrates that it is the fluid motion in the hub that is responsible for the rotation of the sprinkler, not the water in the azimuthal section of the nozzles.

Regardless of nozzle geometry, it appears that the rotation of these large-hub sprinklers can be explained by conservation of angular momentum, until one puts in the numbers. ${ }^{11}$ Having measured the relevant parameters to calculate the various rotational inertias, it turns out that the angular momentum of the water is about $14 \pm 3$ times greater than the angular momentum of the container. Since the mass of the water increases as the container fills, and the water rotates faster near the perimeter compared to the center, the angular momentum of the water could only be determined to an accuracy of one or two significant figures. Nevertheless, there is an order-of-magnitude discrepancy between the water and the container. The situation is much worse for the air sprinklers - the air inside the container would have to rotate $10^{5}$ times faster than the container to conserve angular momentum (this is absurd given that the container rotates as fast as $6900 \mathrm{rpm})$. It is also a fact that, if the air source is turned off while the container is spinning, the container continues to spin for a long time. The rotation of the air inside most likely slows to a halt and then ends up getting dragged in the same direction as the spinning container. What happened to conservation of angular momentum? Clearly there must be other mechanisms at work. Even though these large-hub sprinklers are not the same geometry as the classic Feynman sprinkler (Ref. 1) that prompted these investigations, their behavior provides valuable clues.

To more closely approximate the Feynman sprinkler, the deli container was replaced with a more "conventional" L-shaped sprinkler design. It differs from our original sprinkler model (shown in Fig. 1) in that the arms and elbows can be readily changed to study their effects. The arms are thinwalled, 1/4-in. ID aluminum tubing and attach to a $11 / 2$-in. OD, 3/4-in. ID plastic hub. The exhaust hose couples to the hub, which is secured to the turntable (see Fig. 11).

\section{A FORTUITOUS DISCOVERY}

Out of general curiosity, the operation of the reverse sprinkler was tried without the elbow intake nozzle, as shown in Fig. 11(b). Amazingly enough, it rotated faster than with the elbow in place and still in the reverse direction, although "reverse" has no real meaning without the elbows. The radial symmetry dealt a deathblow to any notion that the rotation was due to missing angular momentum in the fluid surrounding the sprinkler. Partially blocking the intake of the sprinkler arms (with tape) had no effect on the direction of rotation, regardless of which side of the arm was blocked. This curious discovery inspired flipping the elbows of the sprinkler arms in the opposite direction. That too had no effect on the direction of rotation. Doing so only made it look like a rotating "normal" sprinkler, even though it wasn't. Next, the straight sprinkler arms were removed and the hub spun much faster still. Finally, it spun at an incredible $136 \mathrm{~Hz}(8160 \mathrm{rpm})$ upon removal of the copper tube NPT adaptors!

What seemed to be happening was that the two streams of air entering the hub were not simply "smushing" into each other before getting sucked out. Rather, the two streams are somehow being deflected away from each other and 


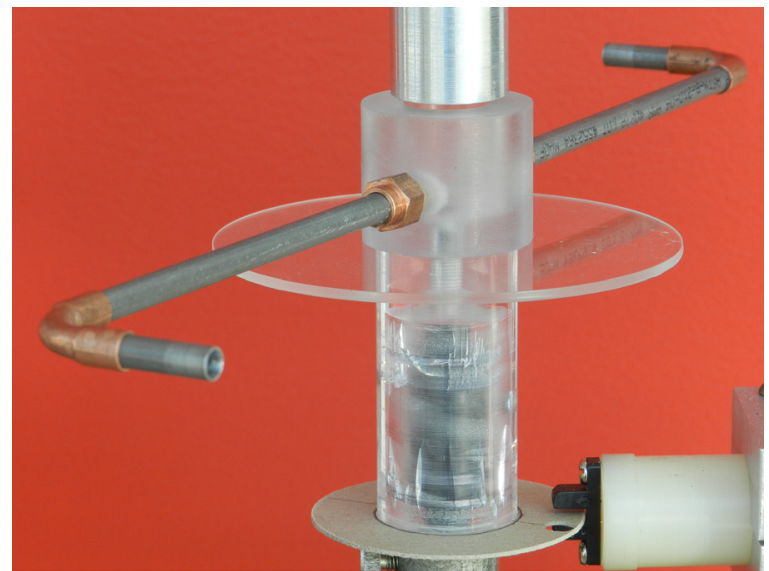

(a)

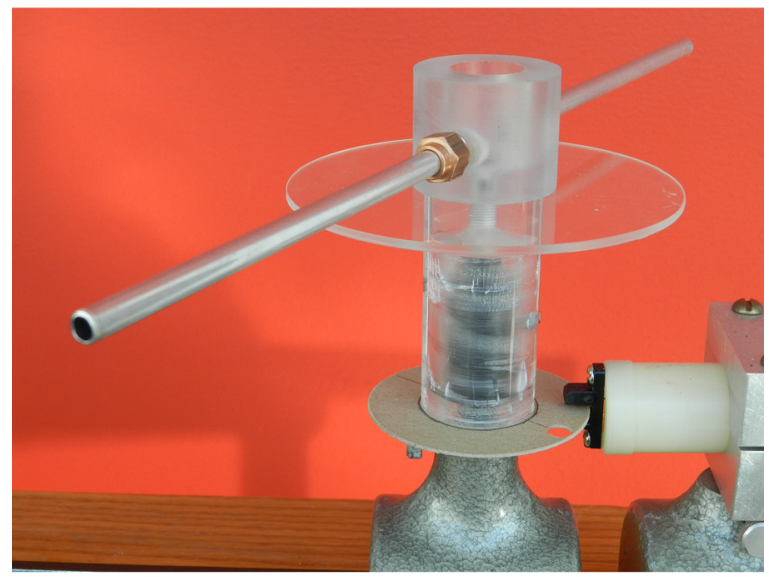

(b)

Fig. 11. L-shaped sprinkler mounted on turntable with and without nozzle.

subsequently form a strong vortex inside the hub. As in the case of the deli container sprinkler, the hub would then rotate in the opposite direction of the vortex. Partially blocking the intakes in different ways had no effect on the motion. ${ }^{12}$ On the other hand, one can affect the direction of rotation if the air inside the hub is already rotating above some threshold speed. For example, if one shuts off the air supply and then turns it back on again while the hub is still rotating, the hub will slow to a halt and reverse its direction of rotation. For example, if the hub is spinning in a CCW direction when the airflow is turned off, it will drag air inside the hub to also spin in a CCW direction. If one then turns the air back on, the rotating air in the hub biases the vortex formation to also be in the CCW direction and the hub, in turn, will slow to a stop and then rotate in the opposite $\mathrm{CW}$ direction. One can continue to toggle the direction of rotation back and forth in this manner as long as the hub is not allowed to slow down below approximately $30 \mathrm{~Hz}(1800 \mathrm{rpm})$. Below that speed it consistently rotates in the same direction. Moreover, since the hub doesn't rotate that fast with the sprinkler arms attached, it is not possible to change the direction of rotation by toggling the air source on and off.

As with the water sprinklers, to test the idea that a strong vortex exists in the hub, a length of $3 / 4$-in.-wide strip of aluminum window screening was rolled up into a cylindrical shape and placed inside the hub to suppress any vortex formation. The incoming air would be forced to scatter through the mesh in a random manner before exiting through the exhaust hose. This did the trick. The sprinkler refused to rotate with the wire mesh in place; it jerks toward the incoming air when the airflow is first turned on, and the opposite transient effect happens when turning the airflow off (although it's not as pronounced because the vacuum motor takes more time to slow down). The geometry of the intake nozzle had absolutely no effect on this behavior. For example, six L-shaped arms with different elbow radii as well an S-shaped configuration were tested (see Fig. 12), and not one of the sprinkler arms rotated with the wire mesh in place.

On the other hand, the sprinkler geometries do behave differently without the wire mesh in place: the larger the radius of curvature in the bend of the intake nozzle, the faster the sprinkler would rotate. Indeed, the rotation rate of the sprinkler using the six L-shaped arms shown in Fig. 12(a) was found to be linearly related to the radius of the elbow; the radii differed by a factor of ten between the smallest and largest, and so did the rotation rates. It is quite possible that turbulence introduced by the elbow has an adverse effect on the vortex formation in the hub. ${ }^{13}$ The S-shaped sprinkler shown in Fig. 11(b) probably generates the least turbulence and it rotated $60 \%$ faster than the fastest L-shaped sprinkler.

It was now evident that the creation of a vortex inside the sprinkler hub was the cause of the steady rotation. However, further investigation was in order to gain insight as to why the direction of the vortex was always the same. Was it because of an asymmetry in the input holes of the hub, how the air streams deflect off the walls of the hub, or how the air streams meet in the hub? Can one control the rotation of the

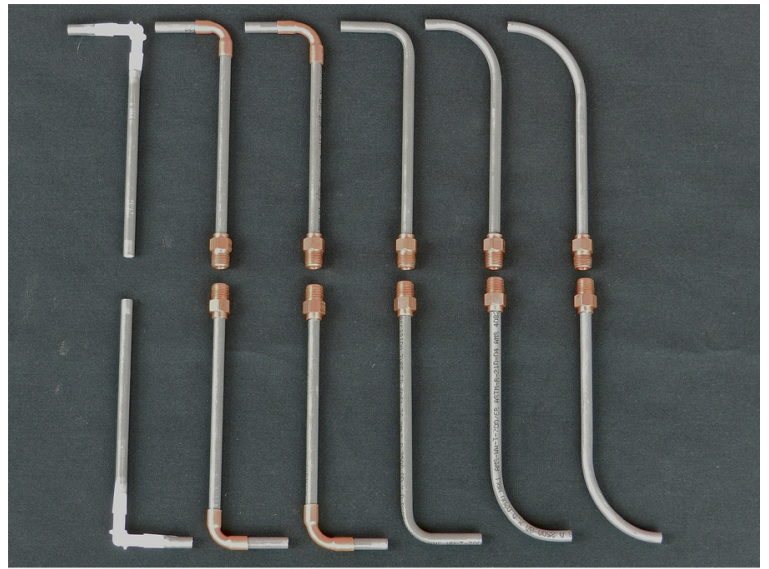

(a)

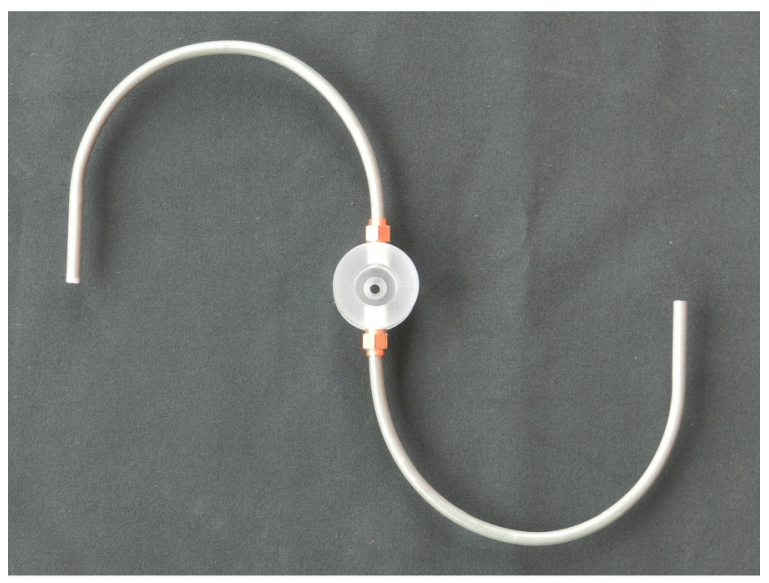

(b)

Fig. 12. Six L-shaped arms and an "S-shaped" sprinkler. 
sprinkler by artificially adding asymmetry? To answer these questions, three experiments were performed: (a) the shape of the input holes inside the hub was altered, (b) the hub wall was modified, and (c) additional input holes and sprinkler arms were added to reconfigure how the air streams meet. The hub alterations are shown in Fig. 13.

Figure 13(a) shows half of the inlet holes blocked by tape. One can readily envision what happens. The tape deflects the incoming airflow to the right, resulting in a $\mathrm{CW}$ vortex rotation, and the impetus on the tape provides a CCW torque on the sprinkler assembly. Indeed, the sprinkler rotated $\mathrm{CCW}$ at a rate of $7.8 \mathrm{~Hz}$ (almost double the rate without the tape). Blocking the opposite side of the hole resulted in opposite (CW) rotation of the sprinkler at the same rate; there was no asymmetry. Asymmetry did rear its head when the tape covered only about $1 / 3$ of the hole-covering the left side of the hole produced a $6.0 \mathrm{~Hz} \mathrm{CCW}$ rotation, while there was no rotation with the tape on the right side. Covering less than $1 / 3$ always resulted in $\mathrm{CCW}$ rotation no matter which side the tape was on, but the rate of rotation was greater with the tape on the left.

Figure 13(b) shows how the hub wall was altered. Brass shim stock was bent into a wedge shape and taped onto the wall. Regardless of the placement and orientation of the wedges, the sprinkler always rotated CCW. Placement and orientation did, however, affect the rate of rotation. For

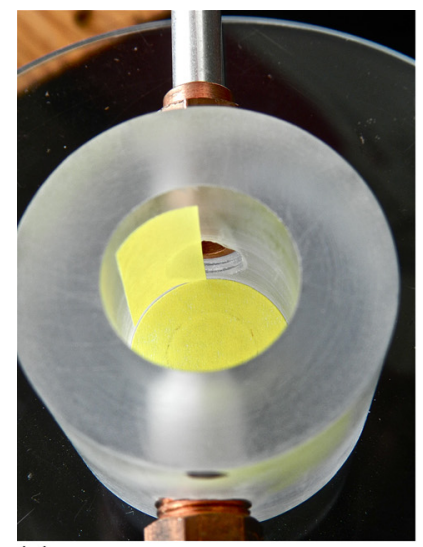

(a)

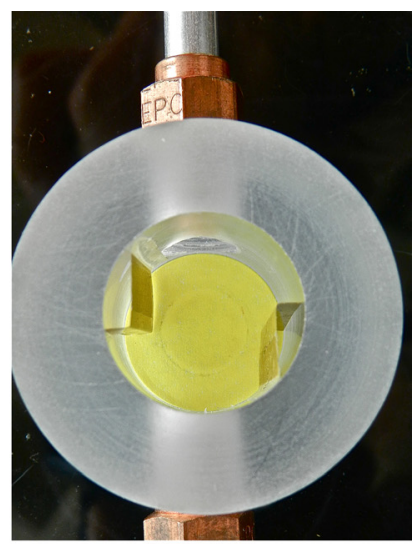

(b) (c)

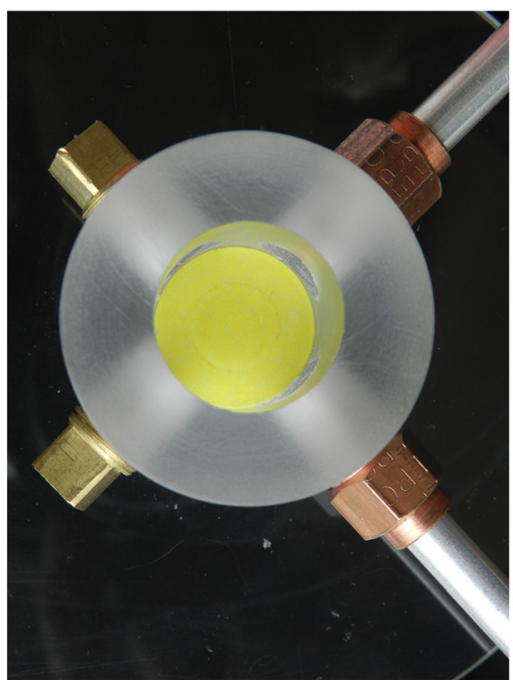

Fig. 13. Sprinkler hub alterations: (a) partially blocking intake holes with tape, (b) wedge-shape on wall of hub, and (c) two additional intake holes to accommodate various arm configurations. example, one would expect the orientation shown in the figure to impede a $\mathrm{CW}$ vortex more than a $\mathrm{CCW}$ vortex and, indeed, the CCW rotation of the sprinkler was only $1.3 \mathrm{~Hz}$ compared to $3.0 \mathrm{~Hz}$ for the opposite orientation of the wedges. From this we conclude that the greater the interaction of the air stream with the inside wall of the hub, the lesser the rate of rotation. However, the interaction is not strong enough to change the direction of rotation. While an asymmetry in the wall is quite observable, the interaction of the two incoming flows dominates the behavior of the sprinkler.

To further investigate the interaction of the two incoming flows, two more holes were drilled and tapped in the plastic hub to accommodate additional sprinkler arms. With the original holes blocked, the two new sprinkler arms caused the sprinkler to turn in the opposite direction at a rate of $3.5 \mathrm{~Hz}$. Since the holes for the new sprinkler arms were machined with the same care and tolerances, this result leads one to conclude that the interaction of the two incoming flows must be very sensitive to the angle between them. The holes were carefully machined but the threads were tapped by hand. It is plausible that their alignment can be off by a fraction of a degree and that that misalignment is enough to break the symmetry. In addition, several configurations were tested, using between one and four arms with different hole combinations (unused holes were plugged). The sprinkler would not rotate with just one arm. Figure 13(c) shows a configuration in which the sprinkler did not rotate, or it rotated either $\mathrm{CW}$ or $\mathrm{CCW}$ at various speeds, all depending on which pair of holes was chosen for the arms.

To completely eliminate the vortex issue, another sprinkler was made. Its hub design does not allow mixing of the air at all inside the hub or the creation of any vortex. The air from each of the four sprinkler arms is simply deflected upward into the exhaust nozzle by an additional elbow, as shown in Fig. 14.

This design can be thought of as an "ideal" sprinkler-the symmetry cannot be broken by the interaction of the airflows. This sprinkler is completely neutral by design and it does not rotate; there is no net torque about the sprinkler's axis of rotation and, since the initial angular momentum is zero, so must the final angular momentum be zero. There is no missing angular momentum in the surrounding fluid. The only motion is an initial jerk toward the incoming air and a small jerk in the opposite direction when the airflow is turned off.

\section{DISCUSSION}

Although the vortex formation in the deli container design was fully anticipated, the vortex in the hub of the L-shaped sprinkler was not. The fact that the hub was carefully fabricated to be as symmetric as possible (within standard machining tolerances) led one to believe that the air coming in from the sprinkler arms would mix randomly before exiting through the exhaust hose. It is now obvious that that was not the case. Rather, this is an unstable dynamic situation and apparently it does not take much to break the symmetry. Instead, the two incoming airflows always deflect off each other and form a strong vortex.

We now come back to the original question: what is responsible for the steady state rotation of the reverse sprinkler? An angular acceleration is the result of a torque, so there must be a force couple (a pair of forces equal in magnitude and oppositely directed) that creates a rotation without translation. Consider the simple apparatus shown in Fig. 15 as an example. 


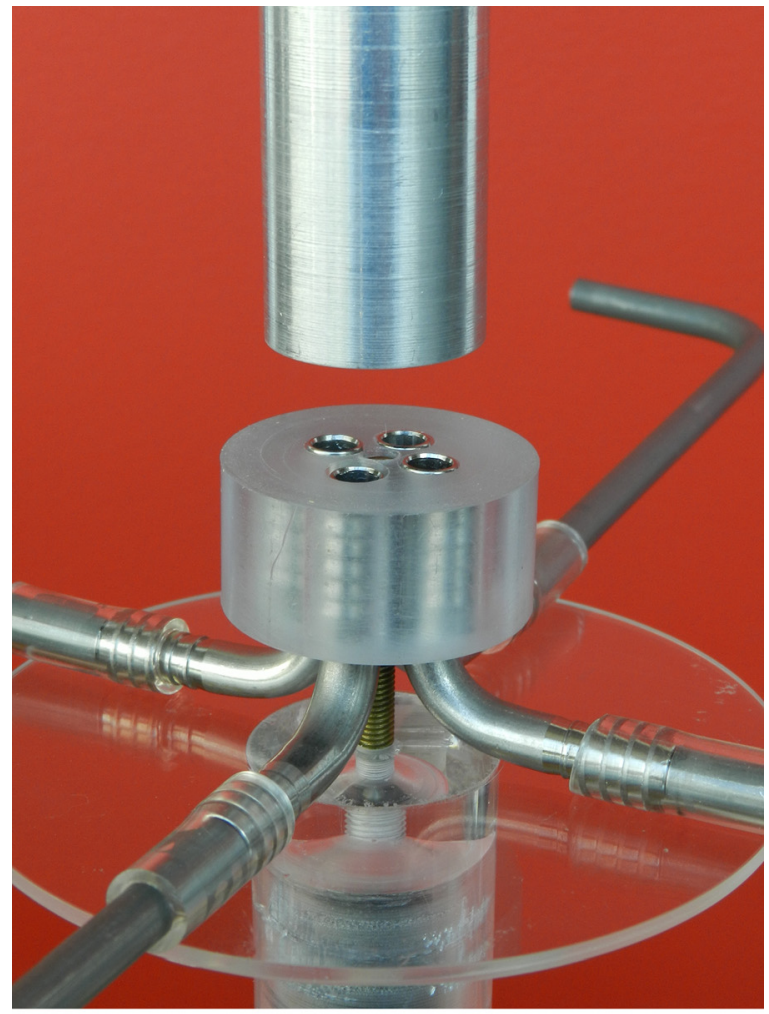

(a)

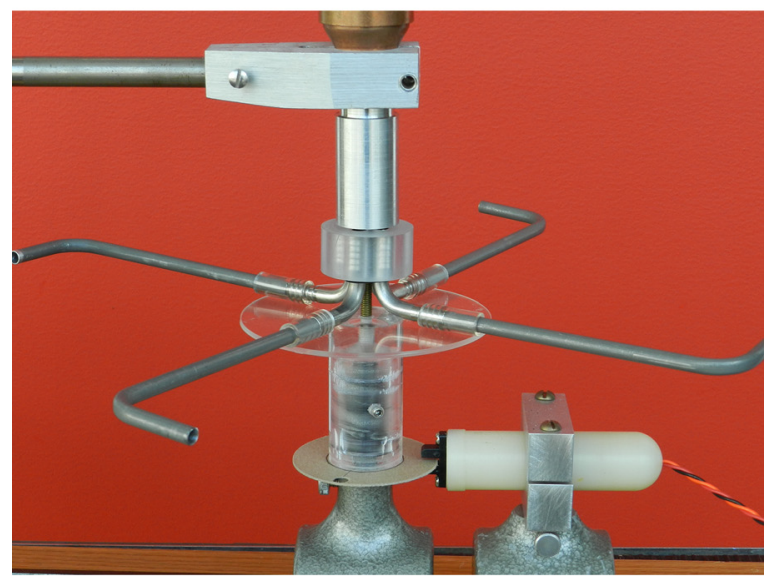

(b)

Fig. 14. Four-arm L-shaped sprinkler with hub designed to eliminate interaction of air jets.

A horizontally oriented disk is made to rotate about its vertical axis. To provide a torque, a string is wound around the hub of the disk. The free end of the string passes over a pulley and is pulled down. This downward force is communicated to the hub by the tension in the string. The tension and the force holding the axle in place constitute the force couple on the disk. The person holding the axle in place and pulling on the string is part of the apparatus. If the person were to do this floating in outer space, (s)he would rotate in the opposite direction of the spinning disk to conserve angular momentum (minus a small amount dissipated by friction). When the person is firmly rooted on the Earth, the entire Earth becomes part of the apparatus and rotates in the opposite direction of the spinning disk to conserve angular momentum. Note that the directions of the original forces initiating all of this are arbitrary but can be redirected mechanically (or through

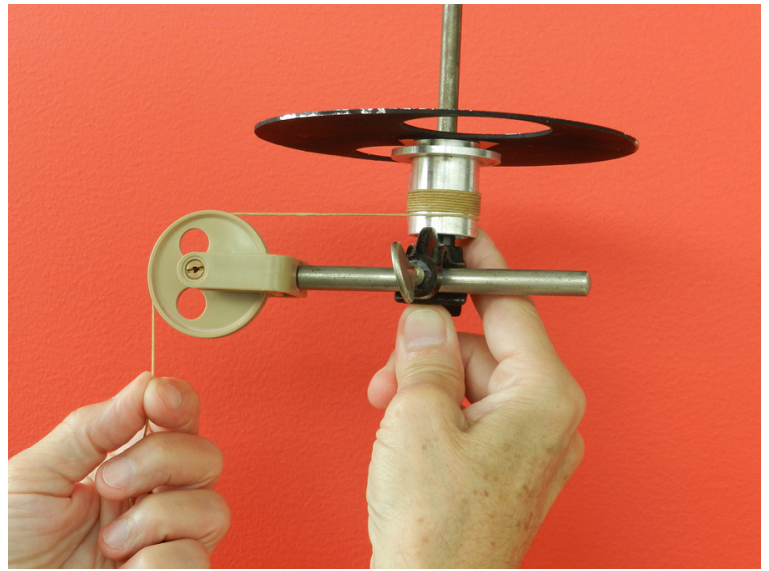

Fig. 15. Example of a force couple providing a torque.

some other interactions) into the final force couple. In the following discussion, the original initiating forces acting on the sprinkler are either gravity or a piston in a pump (whose orientation doesn't matter). These forces become redirected and, together with the force holding the sprinkler axle in place, constitute the force couple on the sprinkler.

Let us first consider the large-hub air sprinkler. It is the pressure difference between the inside of the sprinkler and the outside that is responsible for pushing air into the sprinkler. The force that accelerates air into the sprinkler is given by the product of the cross-sectional area of the intake nozzle $A$ and the pressure difference $\Delta P$, and it is this force that provides the torque about the axis of rotation. The air pump is responsible for $\Delta P$, and thus the force that accelerates the air. The reaction force propels the sprinkler forward toward the incoming air. Furthermore, the pressure difference between the inside of the sprinkler hub and the pump accelerates the air inside the hub, causing it to circulate before it is removed by the pump. Again, it is the reaction to that force that propels the sprinkler in the opposite direction of the circulating air. If the straws in the exhaust hose adapter are removed, allowing for additional rotation of the air inside the hose, the sprinkler rotation speeds increase by approximately $3-4 \%$. This is a very small effect and shows that the rotation of the sprinkler cannot be attributed to rotation of air in the exhaust. With or without the straws, the angular momentum of the circulating air is transferred to the exhaust walls and ultimately to the Earth. The straws simply speed up the transfer process by impeding the circulation more efficiently.

In the case of a large-hub water sprinkler, it is also the pressure difference between the inside and outside of the sprinkler that is responsible for pushing water into the sprinkler. Gravity is responsible for the hydrostatic pressure gradient in the tank as well as pulling the sprinkler down below the surface of the water. The water, being contained by the walls of the tank, is pushed into the sprinkler and it is these pushing forces from opposite walls that constitute the force couple. ${ }^{14}$ The rest of the scenario is the same as the air sprinkler.

In regard to the conventional L-shaped sprinkler, the nozzle geometry is such that it stops the air from moving tangentially with respect to the axis of rotation. Thus, the torque that accelerates the air tangentially into the nozzle is balanced by the torque that stops it-once inside the nozzle, the tangentially moving air is deflected radially inward and the reaction force of the air in turn stops the sprinkler. The force that pushes the air radially in toward the hub, $A \Delta P$, provides no 
torque on the sprinkler arm. However, the continuous generation of a strong vortex inside the hub has to be accompanied by a torque (which itself is a result of the pressure difference), and it is this torque that is responsible for the steady-state rotation of the sprinkler. Suppression of the formation of such a vortex, or a hub geometry that does not allow for vortex formation in the first place, eliminates the steady-state rotation.

Mechanisms for vortex formation can only be speculated on, but undoubtedly are initiated by turbulence and viscous interactions with the walls of the cylindrical hub. Once initiated, vorticity can spread to the rest of the fluid in the interior by the action of viscosity; this was directly observed in the large-hub water sprinklers. The experimentation with the hub inlet holes, such as in Fig. 13(a), show that all the "action" is initiated at the hub entrance orifice and is responsible for breaking the symmetry and deflecting the air in a $\mathrm{CW}$ or $\mathrm{CCW}$ direction to form a vortex. The presence of this vortex further enhances the interaction at the orifice and provides positive feedback. This is evidenced by the fact that if the vortex is not allowed to form (as with the addition of wire mesh), the feedback loop is broken and the small asymmetry at the orifice is not sufficient to cause continuous rotation. The "toggling effect" described earlier is further evidence that the direction of the airflow inside the hub and its interaction with the incoming air is crucial.

Finally, it appears to have been a coincidence that, in all of the original experiments, the vortex formed inside the sprinkler hub happened to rotate in a direction that consistently made the sprinkler turn in the "reverse" direction. Had it rotated in the opposite direction, the story of the reverse sprinkler surely would have unfolded differently. We now understand that a subtle and small asymmetry in the intake holes of the hub has a strong and stable effect on the rotation of our sprinkler. The fact that a (seemingly identical) second set of holes/arms in the sprinkler caused it to rotate in the opposite direction supports this claim. To contend that all the reverse sprinklers having rotated in the reverse direction is a coincidence may raise some eyebrows. However, it turns out that only McGill University, the University of Maryland, and Harvard use the L-shaped sprinkler as a lecture demonstration. ${ }^{15}$ The UMD sprinkler is a close copy of the Harvard design and so a 50/50 chance of behaving similarly is not implausible. As for the McGill sprinkler, Paidoussis and Tétreault-Friend report that theirs generally rotates in the opposite direction to the normal sprinkler, but not always; occasionally it would rotate in the direction of the normal sprinkler, or not at all. ${ }^{16}$ In light of this, the coincidence seems credible.

\section{ACKNOWLEDGMENTS}

The author would like to thank David Morin for thoughtprovoking discussions as well as the anonymous reviewers for their constructive and valuable comments. Their thoughtful critiques and excellent suggestions for additional experiments greatly improved this manuscript.

\footnotetext{
${ }^{\text {a) }}$ Electronic mail: rueckner@fas.harvard.edu

${ }^{1}$ R. P. Feynman, Surely You're Joking Mr. Feynman (Norton, NY, 1985), pp. 63-65.

${ }^{2}$ A. T. Forrester, "Inverse sprinklers: A lesson in the use of a conservation principle," Am. J. Phys. 54(9), 798-799 (1986). Forrester invoked conservation of angular momentum to show that the inverse sprinkler will never rotate but also claims that Feynman's apparatus is governed by different physics because his sprinkler was in a pressurized containment vessel.
}

${ }^{3}$ A. K. Schultz, "Comment on the inverse sprinkler problem," Am. J. Phys. 55(6), 488 (1987). Shultz arrived at the opposite conclusion as Forrester using conservation principles.

${ }^{4}$ A. T. Forrester, "Comments on a letter by A. K. Schultz," Am. J. Phys. 55(6), 488-489 (1987). Forrester claims that the rotation Schultz predicts is only a transient effect.

${ }^{5}$ R. E. Berg and M. R. Collier, "The Feynman inverse sprinkler problem: A demonstration and quantitative analysis," Am. J. Phys. 57(7), 654-657 (1989).

${ }^{6}$ M. R. Collier, R. E. Berg, and R. A. Ferrell, "The Feynman inverse sprinkler problem: A detailed kinematic study," Am. J. Phys. 59(4), 349-355 (1991).

${ }^{7}$ A. Jenkins, "An elementary treatment of the reverse sprinkler," Am. J. Phys. 72(10), 1276-1282 (2004).

${ }^{8}$ Argon, Helium, and the Rare Gases, edited by G. A. Cook, p 196 (Interscience Publishers, NY, 1961), vol. I, p. 196. Although the viscosity variation with temperature is similar for different gases, argon was chosen because its overall viscosity is about $27 \%$ greater than air (or N2). By varying the temperature of the argon between 268 and $313 \mathrm{~K}$, the viscosity varied between 207 and 234 micropoise.

${ }^{9} \mathrm{E}$. Mach, The Science of Mechanics: A Critical and Historical Account of Its Development, translated by T. McCormack, 6th ed. (Open Court Publishing Co., LaSalle Illinois, 1960), p 390. The first German edition is copyright 1883 and the first English translation Open Court edition is 1893.

${ }^{10}$ C. Mungan, "Inverse lawn sprinkler," Phys. Teach. 43, L1-L2 (2005).

${ }^{11}$ The rotational inertia of the 1-quart yogurt container plus copper elbows and washer (for additional ballast) was approximately $1.71 \times 10^{-4} \mathrm{~kg} \cdot \mathrm{m}^{2}$. The rotational inertia of $1 / 2$ liter of water with a 5 -cm radius was calculated to be $6.25 \times 10^{-4} \mathrm{~kg} \cdot \mathrm{m}^{2}$. The rotational inertia of the deli container air sprinkler was approximately $4.15 \times 10^{-5} \mathrm{~kg} \cdot \mathrm{m}^{2}$ (including the turntable doubles this). Treating the air inside as a rotating disk, its rotational inertia is approximately $4.71 \times 10^{-10} \mathrm{~kg} \cdot \mathrm{m}^{2}$.

${ }^{12}$ It has been suggested that the viscous dissipation by flow around the intake nozzle can produce a significant effect resulting in the steady-state torque. For example, S. Dasgupta, P. Mitra, and S. Sengupta report a steady torque by altering the flow pattern around the intake nozzle, in "More on inverse sprinklers," Eur. J. Phys. 11, 311 (1990). They altered the flow by introducing a sheet of cardboard just in front of the nozzle. Although they did not specifically say so, we assume that the cardboard is not part of the sprinkler assembly, but is being held in place by an outside force and therefore they have created a force couple. They enhanced the effect with a sleeve over the nozzle, but that too is assumed to not be part of the sprinkler assembly. We posit that a combination of the Bernoulli and Coanda effects are responsible for the attraction between the nozzle and cardboard or sleeve. We have reproduced their experiments and replicated their results. We then repeated the experiment with the sleeve attached to the nozzle to make it part of the sprinkler assembly, thereby eliminating the force couple. Even though the flow alteration around the intake nozzle was identical, there was no steady torque with this geometry.

${ }^{13}$ The ultimate source of vorticity in the hub has to be from the interaction of the air with the walls through viscous forces. The loss of mechanical energy in the flow is proportional to the friction loss coefficient and is given for commercial pipe fittings in handbooks. For example, for a $90^{\circ}$ elbow, $45^{\circ}$ elbow, and sharp-edged entrance to circular pipe, the coefficient is equal to $0.90,0.42$, and 0.50 , respectively. These values are from W. F. Hughes and J. A. Brighton, Schaum's Fluid Dynamics, 2nd ed. (McGraw-Hill, NY, 1991), p. 121. The assumption here is that friction with the wall produces additional turbulence and that, in turn, has an effect on vortex formation in the hub; there is no way of determining the losses for turbulent flow by purely analytical methods and the effect on vortex formation is entirely speculative.

${ }^{14}$ Jenkins (Ref. 7, p. 1278) points out that having the water acquire a net angular momentum around the sprinkler pivot in the absence of an external torque seems like a violation of Newton's laws only because we are neglecting the movement of the tank itself. Likewise, we argue that since the wall is rooted to the Earth, the Earth moves to conserve angular momentum.

${ }^{15}$ This information is the result of an informal poll taken on the tap-1 listserve. The University of Utah and the U.S. Naval Academy have the sodacan-in-water versions.

${ }^{16}$ M. P. Païdoussis and M. Tétreault-Friend, "Aspirating cantilevers and reverse sprinklers,” Am. J. Phys. 77(4), 349-353 (2009). 